\title{
Attention Profiling Algorithm for Video-Based Lectures
}

\author{
Josef Wachtler and Martin Ebner \\ Graz, University of Technology
}

\begin{abstract}
Due to the fact that students' attention is the most crucial resource in a high-quality course it is from high importance to control and analyze it. This could be done by using the interaction and the communication because they are known as valuable influencing factors of the attention. In this publication we introduce a web-based information system which implements an attention-profiling algorithm for learningvideos as well as live-broadcastings of lectures. For that different methods of interaction are offered and analyzed. The evaluation points out that the attention profiling algorithm delivers realistic values.
\end{abstract}

\section{Introduction}

It is a known fact that students are confronted with a growing quantity of information. Huge amounts of shapes, colors, and text are presented to them in many different ways. Furthermore it is clear that they can handle and process only a limited number of these information at the same time [1. So most of them is filtered out centrally [2]. It has been pointed out that a mechanism known as selective attention is the most crucial resource for human learning [3]. This indicates that managing this attention enhances both, behavioral and neuronal performance 4. 5]

Further important influencing factors of students' attention are both, the interaction and the communication. This means that they should be used in many different forms (e.g. face-to-face, e-mail, live-chat or newsgroups) as well as in all possible directions. So interaction and communication is not only important from the lecturer to the students and vice versa. In addition the so-called studentto-student communication and the interaction of the students with the content itself are key factors of a high-quality learning process especially of a video-based online-course. 6]

Furthermore the mentioned forms of interaction and communication should be used to analyze the attention of the students. So the lecturer should be able to gain more information about the students' understanding of the content. In addition this analysis helps to evaluate if both, the way of presenting and the content itself are suited for the target audience. [7]

So it is clear that human learning processes are strongly depending on the attention of each single student. Furthermore a more detailed knowledge about

P. Zaphiris and A. Ioannou (Eds.): LCT 2014, Part I, LNCS 8523, pp. 358 367, 2014.

(C) Springer International Publishing Switzerland 2014 
the progress of the attention of the students can help to adopt the lecture accordingly. Due to these facts any mechanism helping to analyze students' attention is from high importance.

Based on the mentioned facts regarding students' attention we like to introduce an attention profiling algorithm which operates on interactions. This algorithm is implemented by a web-application which provides different methods of interaction and communication to learning-videos as well as live-broadcastings of lectures. So this could be formulated as the following research-question: implementing an attention profiling algorithm to fully analyze students' attention and evaluating it under realistic conditions.

At first Section 2 presents some related work. After that Section 3 explains the functionalities of the web-application as well as the different parts of the attention profiling algorithm. Finally an evaluation is shown in Section 4 and discussed by Section 5 .

\section{Related Work}

One possible solution to control and to analyze the attention is known as a socalled Audience-Response-System (ARS) which enables the lecturer to present questions to students during the lecture in a standard classroom situation. Furthermore it offers different features of analysis. 8]

Many studies claim that an ARS is a very powerful tool to enhance both, students' attention and participation [9]. So for instance an ARS was compared to other classroom communication methods (e.g. answering questions by cards) by [10. They pointed out that answering questions with an ARS leads to the highest formal participation (100\%). This is also confirmed by [1].

In addition Youtube 1 provides some mechanisms of interactivity to enrich the videos. So for instance it offers the possibility to add questions to videos. However there are not very much features to fully analyze the answers as well as the attention.

\section{Implementation}

This section explains the developed attention profiling algorithm in a detailed way. For that the main functionalities of the web-application called LIVE2 are presented (see Section 3.1) to clarify the operating-context of the algorithm in question. This attention profiling algorithm is divided in two parts. The first one is responsible for a detailed recording of the joined timespans of each single user (see Section [3.2). The second part consists of the calculation of an attentionlevel which is based on the reaction-times of the attendees to the interactions (see Sections 3.3 and 3.4)

\footnotetext{
${ }^{1}$ https://www.youtube.com/video_questions_beta (last accessed December 2013)

2 Short for LIVE Interaction in Virtual learning Environments.
} 


\subsection{Functionalities of LIVE}

As mentioned above $L I V E$ enriches learning-videos as well as live-broadcastings of lectures with different methods of interaction. For that it offers the video or the live-stream to the attendees and if an interaction occurs it displays them as an overlaying dialog (see Figure 1).

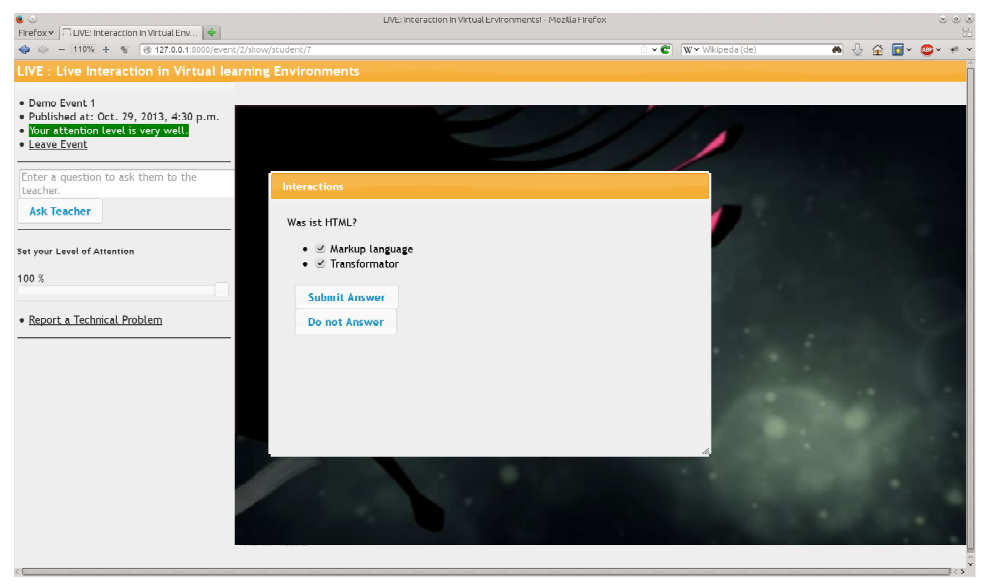

Fig. 1. An interaction occurs during a video

As a summarizing overview the following list presents the most important features of the web-application which implements the developed attention profiling algorithm:

- only available for registered and authenticated users

- different methods of interaction:

- automatically asked questions and captchas 3

- asking questions to the lecturer

- asking text-based questions to the attendees

- multiple-choice questions at pre-defined positions

- reporting technical problems

- many different forms of analysis (e.g. the attention profiling algorithm presented by the following sections)

\subsection{Recording Joined Timespans}

The first part of the attention profiling algorithm implements a detailed recording of the joined timespans of each single attendee. This means that for each

${ }^{3}$ Short for Completely Automated Public Turing Test to Tell Computers and Humans Apart. 
attendee it is possible to say at which time he/she watched which part of the video or of the live-broadcasting.

For that two models are used (see Figure 2). If a user joins an event at the first time and becomes an attendee of this event an instance of the JoinedUser-model is created. Furthermore the method join() of this new instance is called to build the first instance of the History-model. This sets the fields join_relative as well as join_absolute and marks this History-object as active. So a History actually records a joined timespan. For that it holds all relevant data about it in relative as well as in absolute values.

While a user is joined the fields leave_relative and leave_absolute are updated every five seconds. If the attendee leaves the event the according method is called to mark the current History-object as non-active. On a further join it is required to call the according method of his/her JoinedUser-object again which leads to the construction of a further History-object. So it can be seen that there will be a set of History-objects for each joined user where every History-object represents a joined timespan.

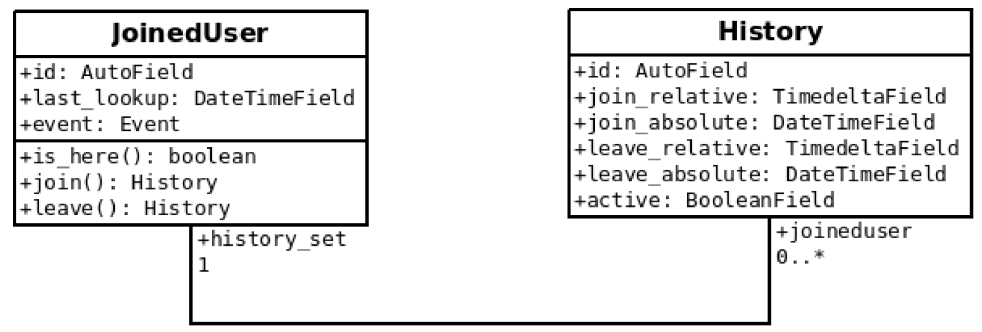

Fig. 2. The models to record the joined timespans of a user (simplified)

With these recordings it is possible to calculate different statistical values as for instance the shortest or the longest joined timespan as well as the average length of the joined timespans. In addition a timeline is drawn for each attendee. It shows the joined timespans by marking them with colored bars.

\subsection{Interactions and Reaction-Times}

As mentioned above the second part of the attention profiling algorithm consists of a calculation of an attention-level which is based on the reaction-times of the attendees to the interactions. For that it is required to log them.

The corresponding models are shown by Figure 3. It can be seen that the logging of the reaction-times works very similar to the recording of the joined timespans presented by the previous section. The interactions are represented by a model with its concrete sub-class models for each possible receiver of an interaction. This model holds a set of CallHistory-models to log every occurrence of the interaction in question. This is required because of the fact that in a video 


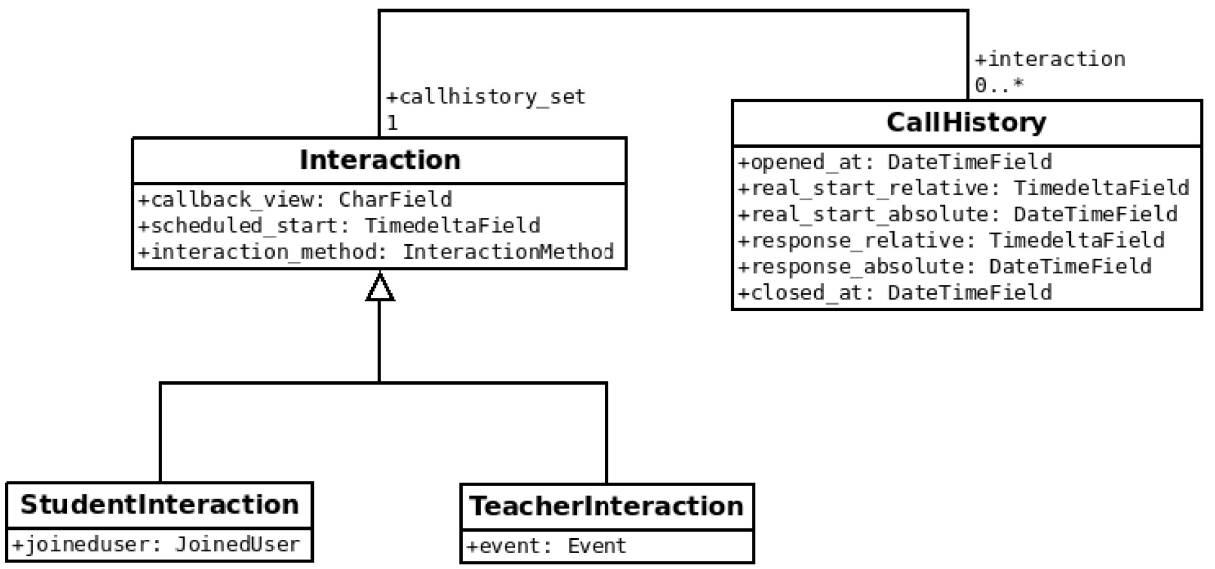

Fig. 3. The models of the interactions and their call-histories (simplified)

the attendee is able to watch it more often than once and so an interaction could also occur not only once.

The CallHistory-model logs all required times to compute the reaction-times of each single call of an interaction in relative and absolute values. For that the fields which contains the real start time and the response time are the most important ones because the difference between them is equal to the reactiontime. The other fields are used if the interaction is missed or not answered. With this values it is possible to state for each call of an interaction how the associated attendee reacted.

\subsection{Calculating the Attention-Level}

With the logged reaction-times presented by the section above now it is possible to calculate an attention-level for each joined timespan of each single attendee. As shown by Figure 4 the calculation is split in three rounds:

1. calculation of an attention-level based on the reaction-times for every call of an interaction (I) related to the joined timespan in question

2. grouping them to attention-levels (AL) of each interaction-methods (IM)

3. generalizing them to an attention-level of a joined timespan

It is clear that round one is the most crucial part of the calculation which operates under the following maxim: if the attendee reacts slower the attentionlevel decreases. Figure 5 visualizes this calculation. It can be seen that it is configured by two parameters which are different for every interaction-method:

1. SUCCESS_UNTIL states the time until an attention-level of $100 \%$ could be reached 


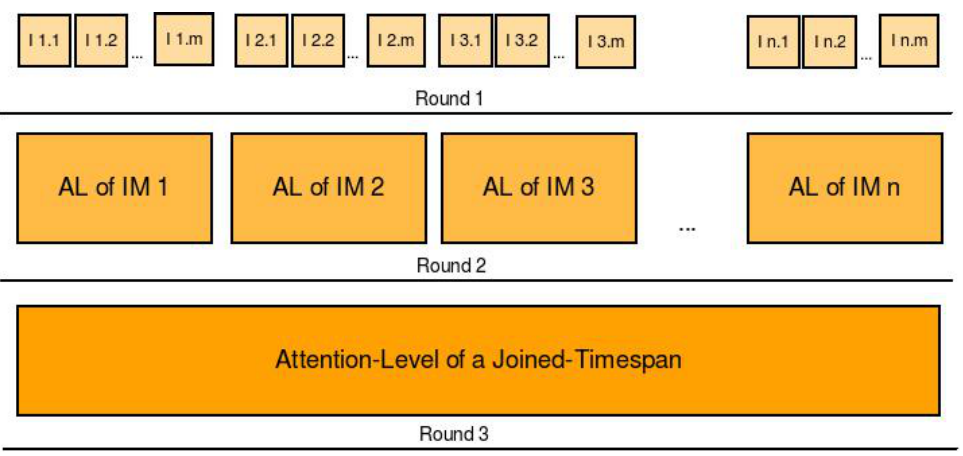

Fig. 4. The calculation of the attention-level is split in three rounds

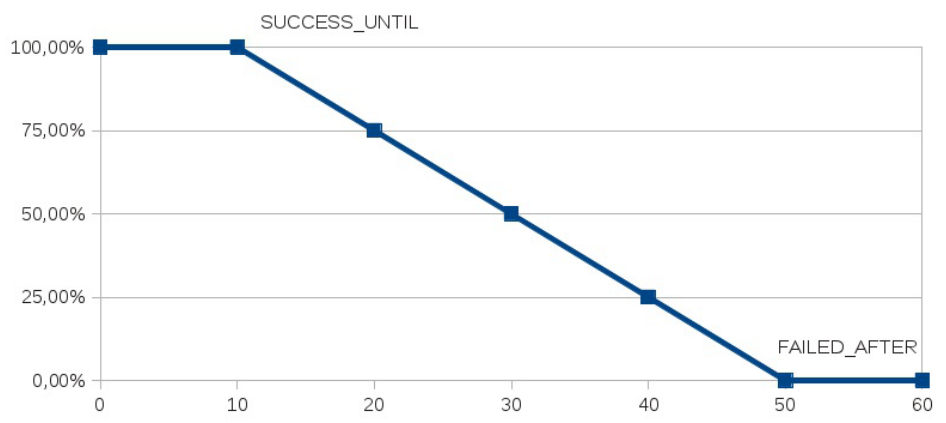

Fig. 5. The calculation of the attention-level of a single interaction is controlled by two parameters

2. FAILED_AFTER indicates after which reaction-time an attention-level of $0 \%$ will be assumed

So the attention-level of the $j$-th interaction of the $i$-th interaction-method is calculated by the following formula if we assume that $t_{i j}$ is the corresponding reaction-time:

$$
f\left(t_{i j}\right)= \begin{cases}100 & \text { if } t_{i j} \leq \text { SUCCESS_UNTIL } \\ 0 & \text { if } t_{i j}>\text { FAILED_AFTER } \\ g\left(t_{i j}\right) & \text { else }\end{cases}
$$

Where $g\left(t_{i j}\right)$ is

$$
g\left(t_{i j}\right)=100-\left(\frac{t_{i j}-\text { SUCCESS_UNTIL }}{\text { FAILED_AFTER }- \text { SUCCESS_UNTIL }} * 100\right)
$$

As mentioned above round two groups the attention-levels of round one to their corresponding interaction-methods. This is done by forming the mean of 
them which leads to this formula where $a_{i}$ will be the attention level of the $i$-th interaction-method and $m_{i}$ the number of its interactions:

$$
a_{i}=\frac{\sum_{j=0}^{m_{i}} f\left(t_{i j}\right)}{m_{i}}
$$

Finally round three takes the attention-level of each interaction-method and again forms the mean over them to receive the final attention-level of a joined timespan:

$$
\text { attention }=\frac{\sum_{i=0}^{n} a_{i}}{n}
$$

\section{Evaluation}

The evaluation of the developed attention profiling algorithm in the context of the described web-application consists of three parts with individual goals:

1. gaining suitable parameters to force the algorithm to deliver realistic values

2. comparing the results of the algorithm with the feedback of the attendees to implement adoptions

3. evaluating the effects of the adoptions

To reach the first goal the web-application is used at two units of the lecture Societal Aspects of Information Technology 4 at Graz University of Technology to offer a live-broadcasting. This lecture consists of several presentations which are hold by guest-lecturers. To gain realistic values for the parameters of the second part of the attention profiling algorithm (see Section 3.4) the recorded reaction-times of the interactions are analyzed. This means that the average reaction-time of them is calculated to place the parameters around this point. Now the algorithm is able to reflect the attention of the attendees in a realistic way.

The second step of the evaluation is performed by using the web-application at the lecture Introduction to Structured Programming 5 at Graz University of Technology. This large freshman-course presents the basics of structured programming in six units. They are offered as live-broadcastings by LIVE. Figure 6 compares the complete number of attendees with the number of active ones. We assume an attendee to be active if he/she watched more than $75 \%$ with an attention-level of at least $50 \%$. It can be seen that the number of active attendees is very low. The feedback provided by the attendees states that they

\footnotetext{
4 https://online.tugraz .at/tug_online/lv .detail? sprache=1\&clvnr=162241

(last accessed December 2012)

5 https://online .tugraz . at/tug_online/lv . detail? sprache=1\&clvnr=162268

(last accessed December 2012)
} 


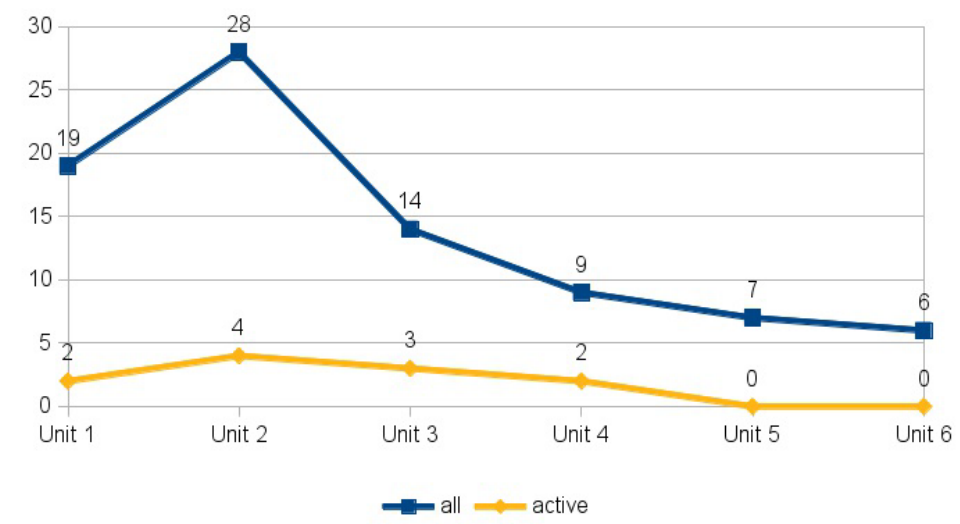

Fig. 6. Comparison of all attendees with active (watched $\geq 75 \%$ and attention-level $\geq$ $50 \%$ ) attendees of the lecture Introduction to Structured Programming

felt very uncomfortable with their attention-level because they assumed a much higher one. Furthermore it was pointed out that it was impossible to answer faster to the interactions presenting difficult questions due to the fact that the live-stream did not stop if an interaction occurs. Additionally it was remarked that the number of interactions should not be very high.

Based on the gained feedback some adoptions are implemented. So for instance the video pauses if an interaction occurs now and it is declared that a lecturer has to pause his/her presentation at the occurrence of an interaction in the case of a live broadcasting. Furthermore the number of interaction is lowered to a maximum of three interactions in a period of ten minutes.

With this adoptions the web-application is used at the lecture Learning in the Net: From possible and feasible things 6 at the Karl-Franzens University of Graz. This lecture explains and discusses different technologies from the field of the so-called new medias in the context of teaching. The content of the lecture is provided by eight videos. Again the number of all attendees is compared to the number of active attendees (see Figure 7). Now these two numbers are not very different. This leads to the conclusion that the attention profiling algorithm is now able to reflect students' attention more realistically.

\section{Discussion}

The evaluation presented by the previous section points out that the selection of the parameters for the calculation of the attention-level is highly sensitive. Their accuracy depends on many different factors as for instance the difficulty of the

\footnotetext{
${ }^{6}$ https://online.uni-graz.at/kfu_online/ lv.detail?cperson_nr=63360\&clvnr=370548 (last accessed December 2013)
} 


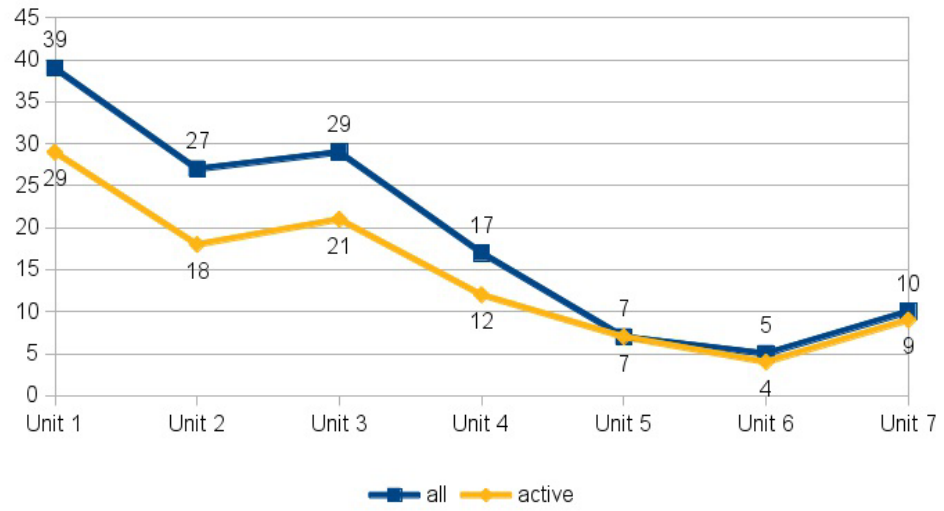

Fig. 7. Comparison of all attendees with active (watched $\geq 75 \%$ and attention-level $\geq$ $50 \%$ ) attendees of the lecture Learning in the Net: From possible and feasible things

questions presented by the interactions as well as the content of the video or the live-broadcasting itself. Furthermore the timespan between the interactions should not be to small. This means that a phase between three and five minutes with no interactions is acceptable.

Furthermore it can be stated that the two parts of the attention profiling algorithm are only powerful in combination due to the fact that they have different goals:

- The detailed recording of all joined timespans for each attendee to state when which part was watched

- The reaction-time based attention-level to indicate how active the corresponding attendee was at a joined timespan

However the correctness of the calculated attention-level has various influencing issues (see above). This leads to the final statement that the attention-level delivers a first overview of the students' attention which could act as a starting point for a more detailed analysis.

\section{Conclusion}

With this publication an attention profiling algorithm is presented to analyze the attention of the students at learning-videos as well as live-broadcastings of lectures. This is done due the fact that students' attention is the most crucial resource in human learning.

This algorithm is implemented by a web-application which enriches the videos or the live-broadcastings with different methods of interaction. In addition the evaluation of the web-application indicates that the attention profiling algorithm delivers realistic values after some adoptions. So the defined research-question is answered finally. 


\section{References}

1. Shiffrin, R.M., Gardner, G.T.: Visual processing capacity and attentional control. Journal of Experimental Psychology 93(1), 72-82 (1972)

2. Moran, J., Desimone, R.: Selective attention gates visual processing in the extrastriate cortex. Science 229, 782-784 (1985)

3. Heinze, H.J., Mangun, G.R., Burchert, W., Hinrichs, H., Scholz, M., Münte, T.F., Gös, A., Scherg, M., Johannes, S., Hundeshagen, H., Gazzaniga, M.S., Hillyard, S.A.: Combined spatial and temporal imaging of brain activity during visual selective attention in humans. Nature 372, 543-546 (1994)

4. Spitzer, H., Desimone, R., Moran, J.: Increased attention enhances both behavioral and neuronal performance. Science 240, 338-340 (1988)

5. Ebner, M., Wachtler, J., Holzinger, A.: Introducing an information system for successful support of selective attention in online courses. In: Stephanidis, C., Antona, M. (eds.) UAHCI 2013, Part III. LNCS, vol. 8011, pp. 153-162. Springer, Heidelberg (2013)

6. Carr-Chellman, A., Duchastel, P.: The ideal online course. British Journal of Educational Technology 31(3), 229-241 (2000)

7. Helmerich, J., Scherer, J.: Interaktion zwischen lehrenden und lernenden in medien unterstützten veranstaltungen. In: Breitner, M.H., Bruns, B., Lehner, F. (eds.) Neue Trends im E-Learning, pp. 197-210. Physica-Verlag HD (2007)

8. Tobin, B.: Audience response systems, stanford university school of medicine (2005), http://med.stanford.edu/irt/edtech/contacts/documents/ 2005-11_AAMC_tobin_audience_response_systems.pdf (online; accessed October 9, 2012)

9. Ebner, M.: Introducing live microblogging: how single presentations can be enhanced by the mass. Journal of Research in Innovative Teaching 2(1), 91-100 (2009)

10. Stowell, J.R., Nelson, J.M.: Benefits of electronic audience response systems on student participation, learning, and emotion. Teaching of Psychology 34(4), 253$258(2007)$

11. Latessa, R., Mouw, D.: Use of an audience response system to augment interactive learning. Family Medicine 37(1), 12-14 (2005) 